Musées, Patrimoine et Culture scientifiques et techniques

172 | 2017

juillet-août 2017

Médiation numérique un site archéologique : à la rencontre entre réalité et virtualité

Florence Caillet-Baraniak

(2) OpenEdition

Journals

Electronic version

URL: http://journals.openedition.org/ocim/1811

DOI: 10.4000/ocim. 1811

ISSN: 2108-646X

Publisher

OCIM

Printed version

Date of publication: 1 July 2017

Number of pages: $12-16$

ISSN: 0994-1908

Electronic reference

Florence Caillet-Baraniak, « Médiation numérique un site archéologique : à la rencontre entre réalité et virtualité ", La Lettre de I'OCIM [Online], 172 | 2017, Online since 01 July 2018, connection on 19 April 2019. URL : http://journals.openedition.org/ocim/1811 ; DOI : 10.4000/ocim.1811

This text was automatically generated on 19 April 2019

Tous droits réservés 


\title{
Médiation numérique un site archéologique : à la rencontre entre réalité et virtualité
}

\author{
Florence Caillet-Baraniak
}

Vue aérienne du Prieuré Saint-Cosme, lors de la campagne de fouille de 2009-2010, dirigée par Bruno Dufä̈, conservateur du patrimoine au Service archéologique du Conseil départemental d'Indre-et-Loire.

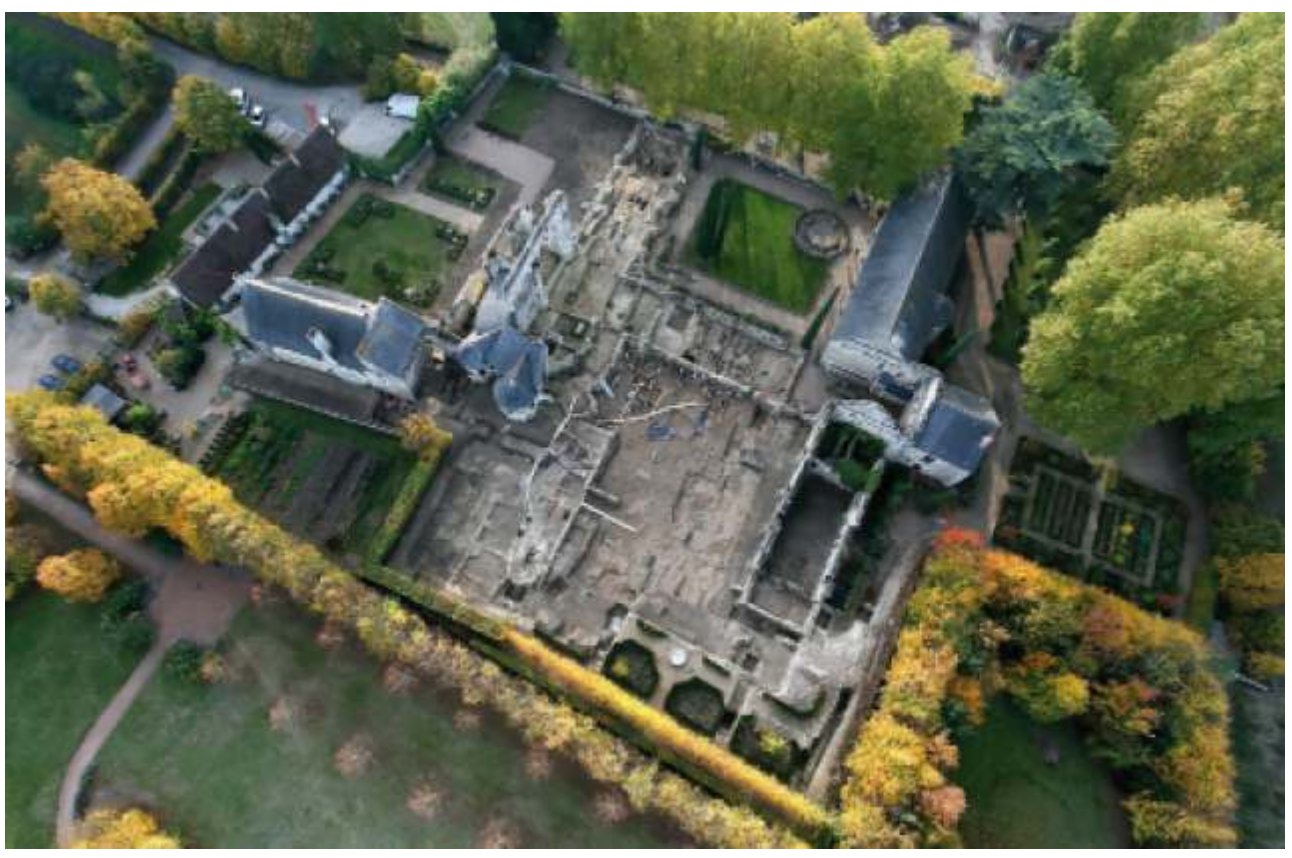

(c) Conseil départemental Indre-et-Loire

Si le numérique est de plus en plus présent en médiation culturelle et sous des formes très variées (applications, numérisation d'informations et de contenus divers, manipulations... ), l'archéologie semble pouvoir en faire un usage particulièrement pertinent : comment, 
en effet, rendre mieux compte de l'aménagement d'un ensemble de bâtiments anciens dont il ne reste que peu de choses à la surface, si ce n'est en permettant au grand public de se l'imaginer et de s'immerger dans sa restitution numérique ? La pertinence de l'utilisation d'une telle technologie n'est pas remise en question. En revanche, la place du numérique dans un lieu patrimonial ne va pas sans s'interroger sur la place des publics et le rôle de la médiation culturelle. Ces interrogations, légitimes et stimulantes, constituent un moyen efficace de mise à distance d'une technologie largement manipulée voire instrumentalisée.

2 Quels sont les usages - espérés et réels - du numérique dans la médiation en archéologie ? Pour quels publics ? C'est ainsi que l'équipe du prieuré Saint-Cosme (Indre-et-Loire) s'est questionnée, face à un outil investi par les archéologues lors des campagnes de fouille du site.

\section{Pourquoi l'archéologie?}

3 En quoi un site archéologique se prête-t-il plus ou mieux à l'utilisation du numérique et à la technologie 3D ? Par définition, celui-ci étant composé de vestiges, la technologie semble requise pour restituer les bâtiments manquants afin de proposer une vision d'ensemble des lieux. Toutefois, il faut se garder de généraliser : cette restitution a un intérêt pour le grand public, nous semble-t-il, uniquement si le site conserve suffisamment de bâtiments pour s'approprier visuellement les lieux mais pas trop non plus, afin que l'effort d'imagination et la virtualité de la 3D puissent encore opérer. Car l'effet recherché avec les restitutions n'est pas d'offrir une visite complètement virtuelle mais bien de faire "travailler" les visiteurs sur la relation entre réalité (l'existant) et virtuel (l'image de synthèse), afin qu'ils obtiennent une image complète, fixe ou évolutive selon les périodes et la plus proche possible de la vérité, du site. Car il est certain que la vision d'une image de synthèse ne peut se suffire à elle-même et doit être confrontée au réel. C'est seulement une fois la confrontation de ces deux mondes, virtuel et réel, réalisée par superposition intellectuelle, que le visiteur pourra se faire une idée (et seulement une idée) de ce que pouvait être le site à son apogée.

\section{L'exemple d'un site archéologique médiéval : la médiation, un enjeu territorial}

4 À $2,5 \mathrm{~km}$ du centre de Tours se situe un monument classé historique en 1946, le prieuré Saint-Cosme, demeure de Ronsard au XVI ${ }^{\mathrm{e}}$ siècle, lieu de sa sépulture à partir de 1585 et propriété du Conseil départemental d'Indre-et-Loire depuis 1951. Longtemps visité uniquement comme maison d'écrivain et jardin de roses, le lieu prend une dimension nouvelle dans les années 2000. En effet, dans le cadre d'un programme de réaménagement paysager et de rénovation de la muséographie autour de la figure du poète, le site a été fouillé par le service archéologique du Département dirigé par Bruno Dufaÿ entre juin 2009 et février 2010. Cette fouille, menée après des sondages réalisés en 2006, a été l'occasion de nombreuses découvertes: les fondations de différents bâtiments du monastère médiéval ont été exhumés ${ }^{1}$, ainsi qu'environ 400 sépultures de religieux et de laïcs². 
Ces découvertes contribuent à la revalorisation de l'histoire du patrimoine local : en transmettant le contenu de ces éléments nouveaux, l'objectif était donc 'interroger le passé du territoire et de mieux le connaître afin de mieux se l'approprier.

Les résultats des études archéologiques ainsi réalisées "deviennent supports 'une médiation territoriale"3. Il s'agit 'un enjeu à ne pas négliger quand on s'adresse au public, et tout particulièrement au public local : ce qu'on transmet aux visiteurs leur appartient et permet une valorisation et une attractivité de ce territoire urbain ; ils en seront 'autant plus prescripteurs auprès de leur famille ou amis qui viennent les visiter dans le Val-deLoire. Pour les médiateurs culturels du lieu, il ne s'agissait donc plus seulement d'évoquer la dernière demeure de Ronsard et le lien avec son jardin de roses mais bien de recontextualiser le poète par rapport au fonctionnement du monastère ainsi que l'histoire plus vaste de ce dernier. L'étendue géographique (2 hectares) et historique du lieu (env. de l'an 1000 à aujourd'hui, en passant par la suppression de la communauté religieuse en 1742) allait changer le discours et les formes de la médiation culturelle sur le site.

La table tactile dans la salle 2 du bâtiment d'accueil, avec sa projection au mur

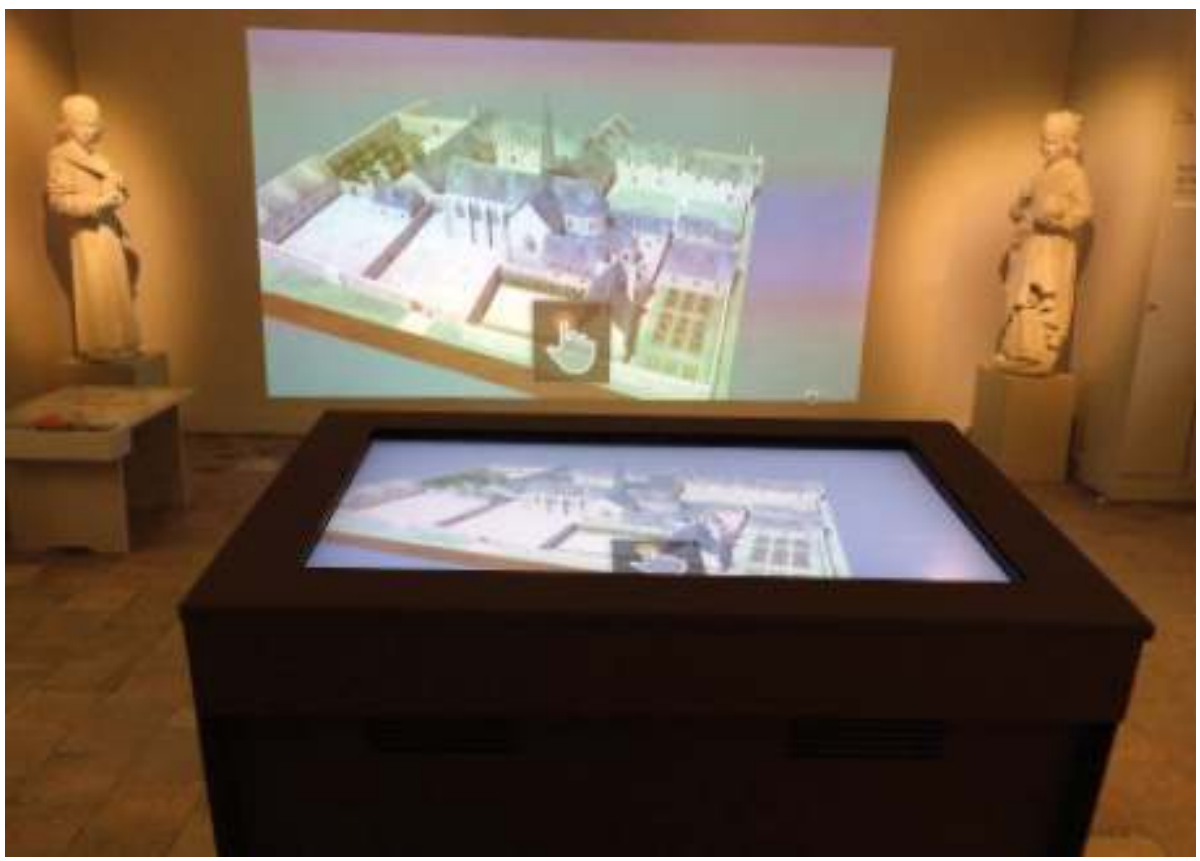

(c) F. Caillet-Baraniak

\section{Exploitation scientifique, transmission et immersion}

L'originalité de l'utilisation numérique dans ce contexte ne tient pas au recours à la modélisation 3D. En effet, de plus en plus, les archéologues font appel à cette modélisation et les destructions récentes du patrimoine mondial de l'Unesco ont fait évoluer très rapidement ces technologies numériques à des fins de mémorisation, de numérisation, de restitution et de reconstitution : l'exemple du travail réalisé par la société Iconem sur le patrimoine syrien est à ce sujet particulièrement éloquent ${ }^{4}$. 
8 Dans le cas du prieuré Saint-Cosme, détruit lui aussi en partie lors de la Seconde Guerre mondiale ${ }^{5}$ l'originalité tient au fait que l'équipe du Service archéologique a utilisé la restitution numérique en cours de fouilles, dès octobre 2009, avant tout comme outil de recherche et non comme outil de transmission. Il s'agissait en effet d'utiliser la technologie pour confirmer ou infirmer les hypothèses des archéologues encore sur le terrain et d'inciter ainsi l'équipe scientifique à se projeter en 3D. C'est ce que Bruno Dufaÿ a appelé la "valeur heuristique des restitutions" : le processus qui pousse à quitter la 2D pour réfléchir en 3D force à évacuer les possibles (et ainsi à faire le choix d'un scénario privilégié) pour tenter de restituer une "vue d'ensemble" homogène d'un bâtiment ou d'un groupe de bâtiments à une époque donnée. Ce processus exige de penser en terme de circulations verticales et de rapport entre les différents bâtiments, il permet aussi de mieux comprendre les structures des constructions. Cette phase de recherche s'est faite en étroite collaboration avec le CNRS, l'université de Bordeaux 3 et la société ArchéoTransfert ${ }^{6}$.

9 Ce n'est que dans un deuxième temps que la restitution a été travaillée pour une transmission et une médiation en direction de tous les publics. Ainsi la phase de restitution au grand public, programmée dans le cadre de la refonte de la scénographie en $2014 \mathrm{a}$ eu l'avantage de s'appuyer sur un modèle abouti. La réflexion autour de son usage de médiation a alors soulevé plusieurs questions :

10 - à qui s'adresse-t-on?

11 - comment transmettre un savoir scientifique (le résultat des fouilles) de manière accessible à tous?

12 - à quel moment et à quel endroit cette restitution doit-elle se faire dans le parcours de visite?

Restitution du 5e état historique retenu par l'équipe scientifique (1570) et proposée aux visiteurs.

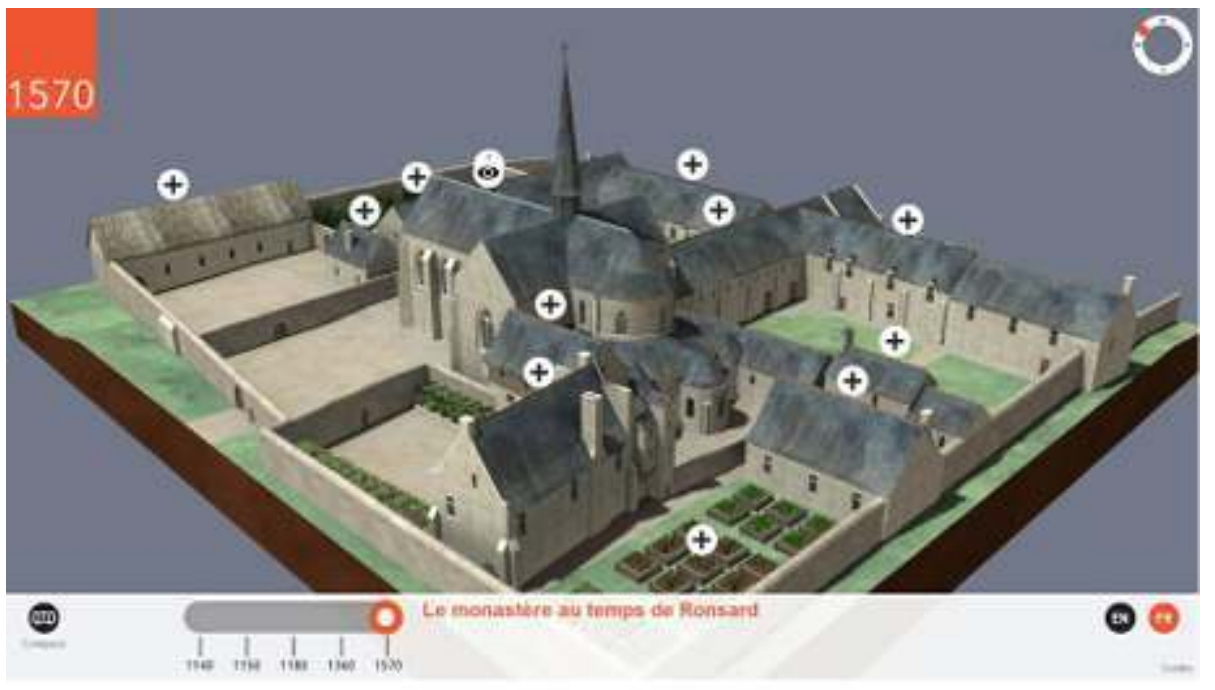

(c) Cent Millions de Pixel/MG Design 
Le visiteur a la possibilité d'accéder à un contenu plus riche, grâce aux pastilles "+" sur l'écran.

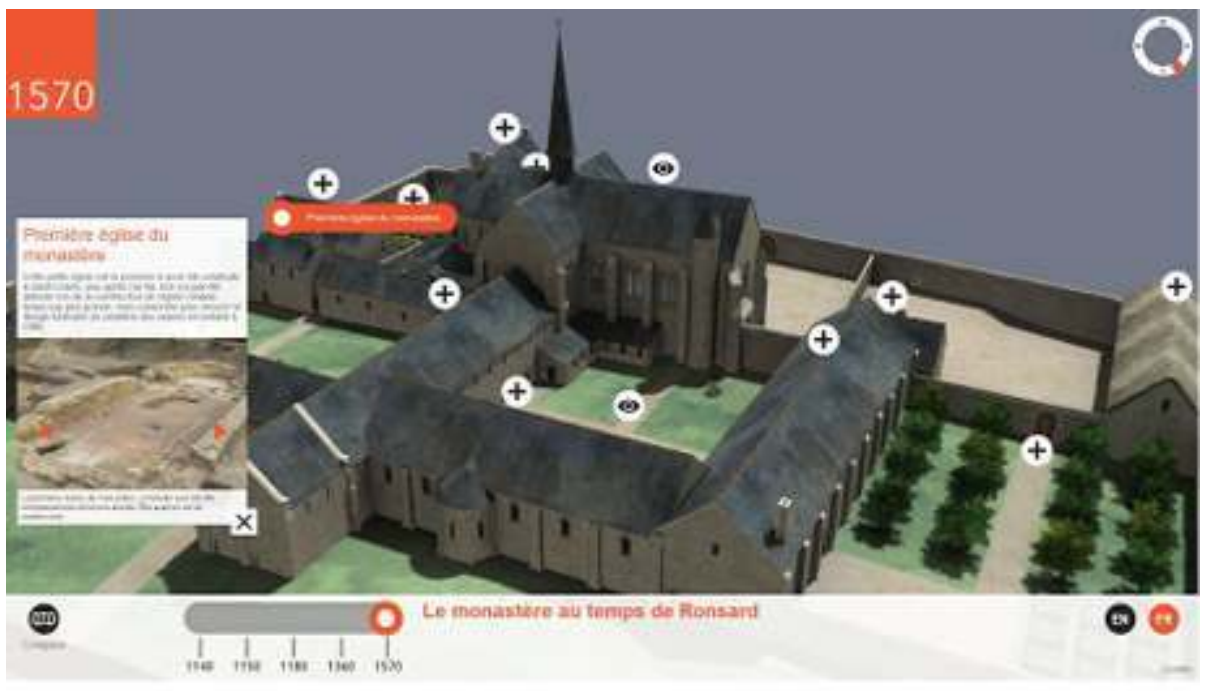

(c) Cent Millions de Pixel/MG Design

\section{Une table tactile, des publics} application 3D à partir de la modélisation déjà réalisée par ArchéoTransfert, tout en continuant de travailler en étroite collaboration avec l'équipe scientifique. Cent Millions de Pixels a reconstitué 5 états historiques du prieuré, la technologie 3D temps réel de MG Design a permis de proposer une application interactive qui facilite la manipulation et l'exploration des bâtiments en extérieur comme en intérieur. Cette application, située dans le bâtiment d'accueil, est accessible aux visiteurs sur une table tactile multitouch de 105 pouces et projetée, à l'aide d'un vidéoprojecteur, au mur de la deuxième salle d'introduction aux lieux ${ }^{7}$. Les deux salles de ce bâtiment d'accueil sont par ailleurs consacrées à l'exposition des objets retrouvés lors des fouilles archéologiques, ainsi qu'au lapidaire ${ }^{8}$. Le circuit de visite permet ensuite aux publics d'accéder à l'extérieur, dans le prieuré proprement dit. évidente pour l'équipe du lieu : sa proximité avec les objets issus des fouilles, sa cohérence avec la présentation générale du site, ont convaincu de la pertinence de cette implantation. Le personnel du prieuré a rapidement compris l'intérêt majeur de cet outil dans une phase introductive des lieux. Il a pour objectifs de :

15 - situer le visiteur par rapport au reste du lieu (grâce à la pastille "vous êtes ici") ;

16 - faciliter la dénomination des bâtiments constitutifs du monastère, ainsi que leur fonction grâce à des pastilles "+" qui se développent avec une courte explication et des visuels de contextualisation (images d'archives et photographies des fouilles); 
20

Depuis sa mise en place en février 2015, plus de 40000 visiteurs ont manipulé ce plan interactif, avec des résultats encourageants par rapport aux objectifs fixés'. Le profil des visiteurs du prieuré Saint-Cosme est multiple et contrasté : de nombreux groupes familiaux (parents, grands-parents, adolescents, jeunes enfants), des groupes de retraités (associations), des groupes scolaires, des individuels aux professions et catégories socioprofessionnelles élevées (enseignants, universitaires et cadres parisiens), des individuels enseignants et étudiants locaux (français et étrangers), quelques visiteurs étrangers (en moyenne $5 \%$ des visiteurs annuels). Le public du champ social est encore faible mais à tendance à augmenter ces dernières années (associations socio-culturelles et visiteurs individuels via Cultures du Cœur). Le pourcentage de visiteurs en situation de handicap, malgré la mise aux normes d'accessibilité du site lors des travaux de 2014, reste faible (1\%).

\section{L'appropriation de l'outil par les visiteurs : quels constats ?}

22 La présence d'un outil numérique, présence forte puisqu'il s'agit d'une grande table installée au cœur des collections archéologiques, est accueillie avec beaucoup d'intérêt car elle invite à l'interactivité donc à l'action, là où les objets des fouilles réclament observation et lecture des cartels. Si l'équipe était persuadée de la facilité d'appropriation par les jeunes générations, elle a été surprise par l'aisance avec laquelle les adultes, actifs et retraités, manipulaient l'application. Il s'agit ici de faire un constat simple : en 2017, les actifs et retraités qui fréquentent les lieux patrimoniaux ont apprivoisé les nouvelles technologies, autrefois pensées comme l'apanage des jeunes, et l'écart entre générations semble s'estomper. Lors des entretiens informels menés avec les visiteurs actifs et retraités, l'importance accordée au maintien du lien familial et intergénérationnel est fréquemment évoquée pour expliquer une certaine familiarité avec les outils numériques et en particulier avec la tactilité de l'écran.

23 Nous ne reviendrons pas ici sur les études faites à partir de l'observation des comportements des visiteurs face au multimédia et en particulier les études générationnelles et de genre. En revanche, nous constatons que l'utilisation d'une application sur grand écran, avec un mode d'emploi simple et accessible et une manipulation intuitive ne constitue pas ici une "frontière numérique" entre les générations ; nous avons pu au contraire observer que ces dernières se réunissent et échangent d'autant plus autour du contenu de la table tactile qu'elles sont toutes capables d'interagir avec elle.

Certains visiteurs, une minorité toutefois, marquent un réel rejet quant à l'utilisation du numérique dans un site patrimonial : ils le pensent trop complexe d'utilisation et parfois inutile. En effet, l'irruption d'un outil, déjà largement présent dans leur quotidien, au cœur d'un monument historique et conservé comme tel, les bouscule dans leur représentation d'un lieu préservé du monde et des progrès technologiques. La crainte de ne pas être "compétent" dans la manipulation de la table tactile les conforte dans la posture du visiteur habitué à n'utiliser que principalement le sens de la vue et qui n'envisage pas d'aller chercher l'information avec le toucher. Ces publics, qui préfèrent 
dans un premier temps une médiation plus traditionnelle, orale ou écrite, gagnent pourtant en confiance quand ils ont été initiés à la manipulation par un médiateur ou par d'autres visiteurs avant eux. L'interaction entre visiteurs prend alors, avec la présence d'une table tactile, une dimension particulière : la possibilité de réunir autour de la table plusieurs personnes (jusqu'à cinq en moyenne) mais la contrainte initiale d'un seul "manipulateur" (seuls deux doigts d'une même personne sont reconnus par l'écran) rend non seulement possible mais presque obligatoire l'instauration d'un dialogue entre les observateurs qui peuvent proposer une manipulation et l'acteur qui manipule l'écran. La présence de la projection murale renforce l'aspect collectif de cette application numérique.

La table tactile, inclinée vers l'avant, est rendue accessible à une hauteur d'enfant à partir de 5/6 ans. Pour les médiateurs, la table est rapidement accueillie comme un outil didactique, qui sert de point de départ à l'exploration du prieuré, notamment avec les groupes scolaires. En effet, la possibilité de manipuler, de "rentrer" dans les lieux restitués à l'époque médiévale et des Temps Modernes, tout en offrant la possibilité à tout le groupe (25 à plus de 30 élèves parfois) de voir le contenu de la table grâce à la projection murale, représente un avantage certain par rapport à la maquette sous vitrine cloche exposée précédemment. Ainsi, ce n'est plus le groupe scolaire qui tourne autour de la maquette, mais la maquette qui se tourne pour offrir ses différentes faces à l'étude. Chaque bâtiment est nommé, repéré et son attribution est rendue plus accessible par une œuvre ou un schéma qui clarifie sa fonction au Moyen Âge.

La comparaison entre deux époques permet de mieux comprendre l'évolution et les modifications des bâtiments.

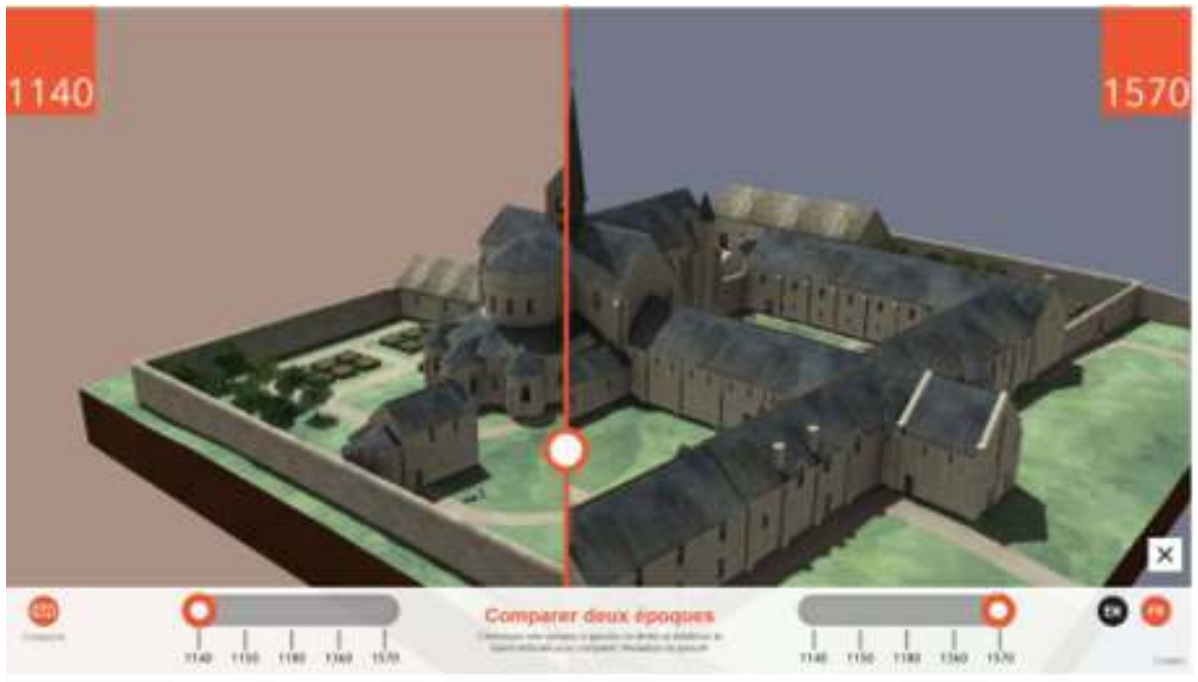

\section{L'appropriation des contenus : des objectifs atteints, le rôle du médiateur confirmé}

L'objectif initial, celui de transmettre des résultats scientifiques de manière accessible au plus grand nombre, sans trahir la réalité de ces résultats ni leur complexité, semble atteint. Les visiteurs comprennent l'organisation du monastère au Moyen Âge et à la 
Renaissance, selon les hypothèses émises par les archéologues suites aux fouilles et à l'étude des artefacts retrouvés. Toutefois, l'objectif n'est pas complètement atteint si le visiteur ne parvient pas à faire le lien entre la maquette virtuelle et les vestiges qu'il va ensuite découvrir dans le prieuré. Le rôle du médiateur est à ce niveau très important : c'est lui qui va insister dans sa présentation de la maquette virtuelle sur les éléments encore visibles aujourd'hui et lors de sa présentation des vestiges, il pourra convoquer à nouveau à l'esprit des visiteurs l'image virtuelle qui correspond à ce qu'ils regardent. Ainsi le va-et-vient entre réel et virtuel nécessite une médiation humaine qui va créer les liens entre bâtiments en pierre et restitution numérique. Et la solution de la réalité augmentée, si elle était financièrement possible, ne serait pas un substitut équivalent à ce rôle central du médiateur dans la mise à disposition d'un outil numérique au public.

Manipulation de la table tactile lors d'une visite en famille.

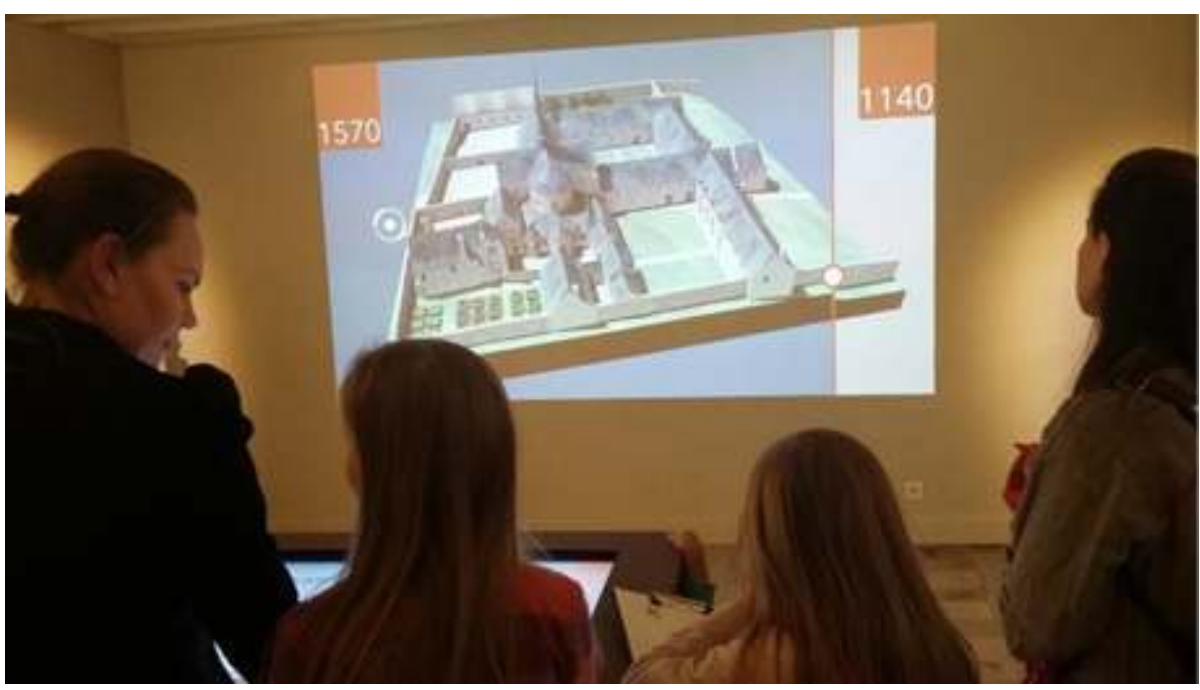

(c) F. Caillet-Baraniak

Une immersion virtuelle dans le cloître en 1360, à l'apogée du prieuré

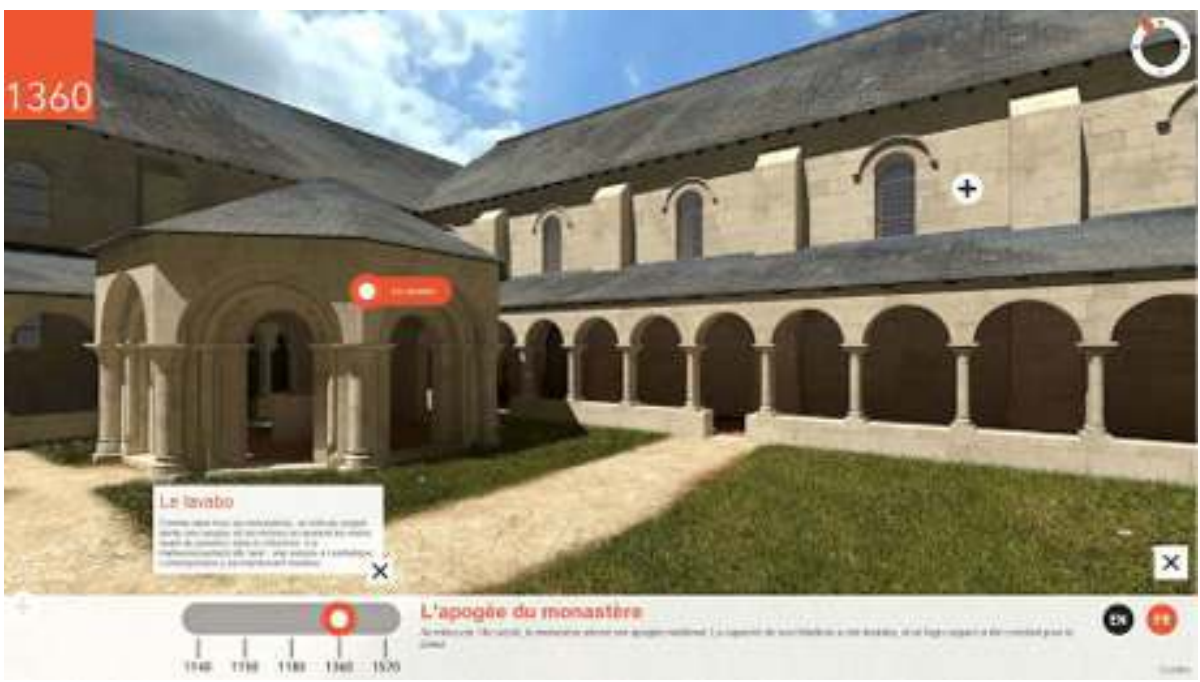

(c) Cent Millions de Pixel/MG Design 


\section{Limites et perspectives : un outil perfectible, une médiation à affiner}

Pour des raisons essentiellement financières, la maquette 3D numérique n'est pas contextualisée : le contexte géographique pourtant essentiel à la compréhension du lieu n'a pas pu être ajouté. Il manque donc la localisation de la Loire au nord, aujourd'hui invisible depuis le prieuré en raison de la construction de la digue, mais qui a bien sûr un intérêt stratégique et économique dans l'implantation du prieuré à cet endroit. De plus, au fil des siècles, un bras du fleuve, qui passait devant le côté sud du prieuré et qui en faisait donc une île sur la Loire, a disparu, expliquant ainsi l'ancienne appellation de "Saint-Cosme-en-l'île" donnée au lieu dans les archives. Ces éléments, qui sont fondamentaux dans la lecture du paysage et l'explication du fonctionnement du monastère, ne sont donnés aujourd'hui aux publics que par des outils de médiation plus traditionnels : panneaux avec cartes topographiques évolutives, textes et médiation orale lors des visites guidées.

De plus, un des publics mis de côté par cette table interactive est évidemment le public en situation de handicap visuel et plus particulièrement les personnes aveugles. L'effort demandé aux visiteurs voyants de garder en mémoire les éléments de restitution au moment de la visite in situ est impossible à demander à ce public. Il est donc impératif d'envisager un plan tactile, qui pourra prendre diverses formes : impression tactile d'un plan simplifié, transportable par les personnes in situ ou/et plan tactile fixe dans les salles du bâtiment d'accueil, à proximité du plan interactif. La question de la représentation des cinq états historiques du prieuré ne serait pas résolue pour autant et nécessiterait la création de cinq maquettes différentes.

Enfin, l'évidence de la place de la table tactile au sein du parcours est réinterrogée à la lumière des expériences de visiteurs. En effet, sa localisation en tout début de visite, avant même l'appréhension physique du lieu, réclame, de la part du visiteur, un effort important d'imagination puis de mémorisation. Il est possible que la manipulation de l'application, pertinente comme introduction du lieu, le soit aussi comme synthèse de la visite mais suppose que le visiteur fasse la démarche individuelle de retourner dans les premières salles pour consulter de nouveau la table tactile. Pour les visiteurs en groupe, le médiateur doit rappeler aux publics cette possibilité afin de se remémorer les restitutions des bâtiments disparus et ainsi compléter la compréhension globale des lieux.

\section{Bilan non exhaustif}

Les mutations numériques que vivent aujourd'hui les médiations culturelles et le secteur de la recherche sont au cœur des réflexions que doivent intégrer les professionnels de la culture afin de ne pas se laisser submerger par les injonctions politiques, économiques et sociétales. Depuis les années 2000, les colloques internationaux sur la question se multiplient, favorisent les échanges de bonnes pratiques et des expériences. L'expérience de terrain relatée ci-dessus nous conduit à dégager quelques principes :

31 - le caractère indispensable des études de comportement des publics au sein de nos institutions, si évolutifs soient-ils ; 
32 - le caractère perfectible de nos dispositifs numériques et le champ des possibles qu'il faut savoir garder grand ouvert en raison de l'extrême rapidité des innovations techniques et technologiques et de la forte capacité d'adaptation des publics ;

3з - la richesse du travail interdisciplinaire et sa grande compatibilité avec les attentes du public ;

34 - le rôle irremplaçable et complémentaire de l'humain dans la construction de la médiation culturelle et dans la transmission, ne serait-ce que - et surtout - de la passion, sentiment proprement et exclusivement humain. Gageons que ce dernier principe, lui, soit une constante.

\section{BIBLIOGRAPHY}

Dufaÿ, B. La modélisation 3D de grands ensembles monumentaux de la restitution au public à la recherche scientifique, Archeologia e calcolatori, supplemento 5, 2014, pp. 149-163.

Mahoudeau, J. L'archéologie aux défis de la médiation culturelle hypermédia, in Colloque international Mutations des industries de la culture, de l'information et de la communication, septembre 2006

L'expérience de la visite et la place du numérique. Bibliographie commentée. Stage organisé par l'Ocim à Bordeaux, les 21 et 22 mars 2013.

Deux pouces et des neurones : les cultures juvéniles de l'ère médiatique à l'ère numérique. La Documentation française, Paris, 2014.

Site du Prieuré Saint-Cosme : http://www.prieure-ronsard.fr Site de ArchéoTransfert : http://www.archeotransfert.cnrs.fr

\section{NOTES}

1. Il s'agit notamment des vestiges d'une église primitive de l'an mil et des bâtiments distribués par le cloître comme la salle capitulaire et le dortoir.

2. L'étude de ces sépultures est en cours, certains crânes trépanés font l'objet d'une recherche particulière.

3. Savary, X. Les supports pédagogiques pour la médiation en archéologie, Les nouvelles de l'archéologie, n¹22, 2010, pp. 5-10.

4. Base de données "Syrian Heritage" réalisée par Iconem : http://iconem.com/fr/ heritage_syrien

5. En mai et juin 1944, le prieuré est victime des lourds bombardements alliés qui visent le pont ferroviaire voisin. Le sous-prieuré, l'infirmerie, l'hôtellerie, le porche d'entrée et le réfectoire largement restauré en 1947 - sont fortement endommagés.

6. Pour le détail de cette collaboration et ses enjeux, voir Dufaÿ, B. et Mora, P. Les restitutions 3D du prieuré Saint-Cosme (La Riche, Indre-et-Loire). La modélisation d'un ensemble complexe à plusieurs 
phases chronologiques. Actes du colloque Virtual Restrospect 2013, Archéovision 6, éditions Ausonius, Bordeaux, p. 138.

7. Dimensions de la projection murale : $180 \times 230 \mathrm{~cm}$

8. Il s'agit de pots en terre cuite, utilisés lors des cérémonies funéraires, des objets retrouvés dans les sépultures, le retable en pierre de l'autel, ainsi que des statues.

9. Une enquête de satisfaction a été réalisée auprès des visiteurs en 2015. Les conclusions fournies dans ce texte sont les résultats d'une observation des comportements des publics en 2016 ainsi que d'échanges avec eux en début, en cours et en fin de visite.

INDEX

Mots-clés: Numérique, 3D, archéologie

\section{AUTHOR}

\section{FLORENCE CAILLET-BARANIAK}

responsable adjoint du prieuré Saint-Cosme

fcaillet@departement-touraine.fr 